\title{
Images anciennes et nouvelles de Blaise Pascal
}

Souvenir de l'exposition organisée par le CIBP

\section{Yves Morvan}

\section{(2) OpenEdition}

\section{Journals}

Édition électronique

URL : http://journals.openedition.org/ccibp/628

DOI : $10.4000 /$ ccibp.628

ISSN : 2493-7460

Éditeur

Centre international Blaise Pascal

\section{Édition imprimée}

Date de publication : 7 janvier 1991

Pagination : 17-28

ISSN : 0249-6674

Référence électronique

Yves Morvan, «Images anciennes et nouvelles de Blaise Pascal », Courrier du Centre international Blaise Pascal [En ligne], 13 | 1991, mis en ligne le 08 janvier 2016, consulté le 15 septembre 2020. URL: http://journals.openedition.org/ccibp/628

Ce document a été généré automatiquement le 15 septembre 2020

Centre international Blaise Pascal 


\section{Images anciennes et nouvelles de Blaise Pascal}

Souvenir de l'exposition organisée par le CIBP

Yves Morvan

1 Textes et composition des planches dus à Yves Morvan en collaboration avec le Club Micro-Data et le CRDP de Clermont-Ferrand et présentée à l'Espace municipal Pierre Laporte à l'occasion du Colloque « Droit et Pensée politique autour de Pascal » Octobre 1990.

\section{Planche I}

On recherche... 
Planche I On recherche...

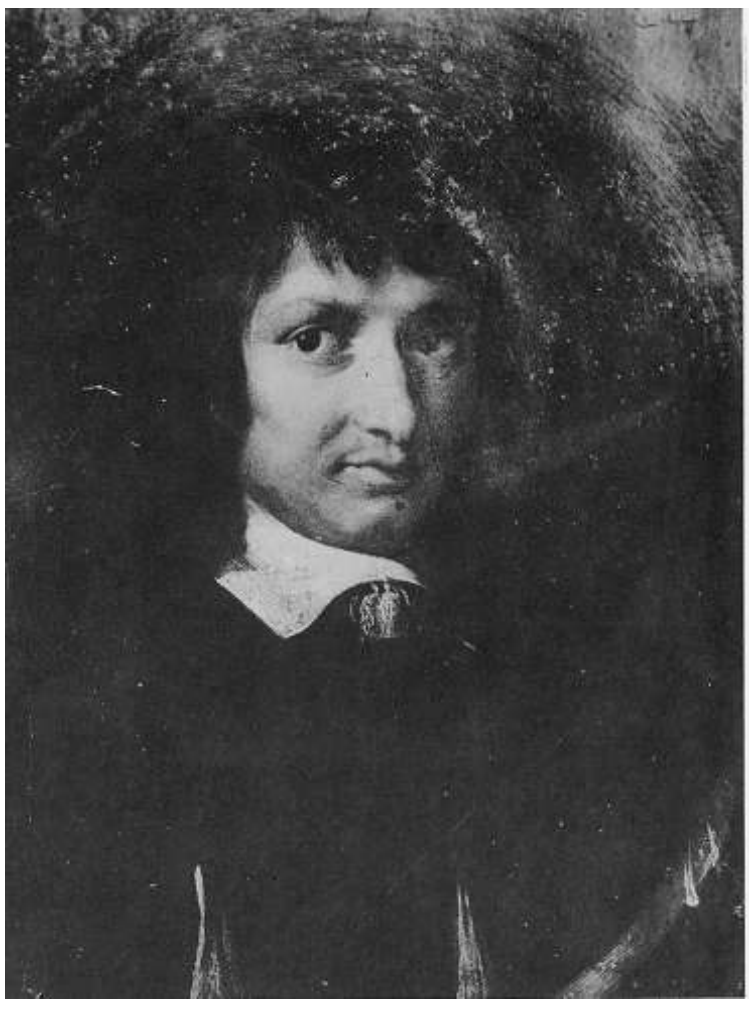

«De son physique proprement dit : stature, physionomie, teint, allure générale, que savons-nous? Au fond très peu de chose. À part la jolie sanguine de Domat et le masque mortuaire, il ne nous reste rien de sûr pour suggérer l'image de ce qu'il fut. »

\section{Planche II}

\section{Le dessin de Domat}




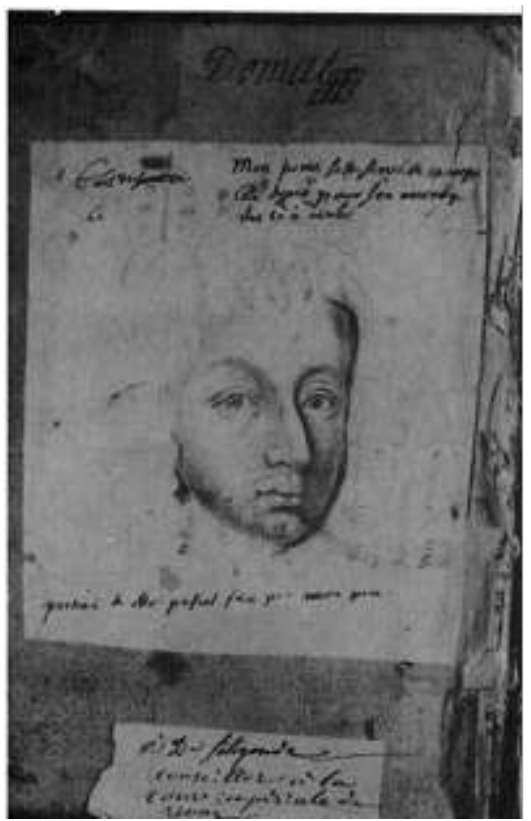

Bibliothèque nationale de France, Paris

Ce portrait au crayon rouge représente Blaise Pascal adolescent (entre 14 et 19 ans). Il a été dessiné par son ami Jean Domat, célèbre jurisconsulte clermontois. Blaise Pascal habite Paris depuis la fin de 1631. Jean Domat y arrive en octobre 1635 pour entrer au collège de Clermont (aujourd'hui Lycée Louis Le Grand) où il apprit le dessin pendant ses études.

En 1639, Pascal est à la veille de quitter Paris pour la Normandie. Il a seize ans et Domat quatorze, dont quatre années d'étude du dessin. "Ne pourrait-on supposer, note M. Dorival (Revue du Louvre $\left.\mathrm{n}^{\circ} 2,1962\right)$, qu'au moment d'être séparé de son ami sur le point de partir pour la Normandie, l'un des adolescents aurait alors " tiré " l'autre, comme on disait alors ?». M. Dorival a ensuite changé d'avis et affirmé que le dessin avait été exécuté après la mort de Pascal. Pour nous, ce portrait a bien été dessiné du vivant de Pascal et d'après nature ${ }^{1}$

4 Bernard Dorival soutient qu'il a été copié - et Pascal rajeuni de vingt ans "par maladresse»! - sur le portrait posthume peint par Quesnel d'après le masque mortuaire.

5 Or, l'étude comparative du dessin de Domat avec la peinture de Quesnel établit l'impossibilité d'une copie de l'un sur l'autre. Sont différents en effet: l'angle de vue, l'éclairage, la chevelure, la courbure du nez, le front, les yeux plus ouverts, l'écartement des sourcils et leur implantation, les paupières, la couleur des yeux, etc.

6 Tant de différences ne peuvent s'expliquer par la prétendue maladresse de Domat.

7 Cette effigie pascalienne est l'œuvre d'un amateur averti : exécutée avec une précision appliquée, elle est d'une ressemblance qui n'étant contestée par personne (pas même M. Dorival), lui confère une valeur incommensurable.

8 Comme en outre, ce dessin de Domat offre plus de ressemblances avec le masque mortuaire que n'en présente le portrait par Quesnel, on peut affirmer que le portrait dessiné par Domat est plus ressemblant à Pascal que celui peint par Quesnel. 
9 Après la mort de Jean Domat, son fils Gilbert colla ce dessin sur le plat intérieur d'un ouvrage de droit dont son père s'était servi et l'annota pour signaler l'identité du modèle.

10 Ce "précieux volume, relique de Domat servant de reliquaire à la relique de Pascal» (Albert Ojardias) ne fut retrouvé qu'au XIXe siècle dans une famille de magistrats de Riom. Il se trouve actuellement à la Bibliothèque Nationale de Paris. Il a été exposé par le CIBP à Clermont Ferrand en 1981.

Le jurisconsulte Jean Domat

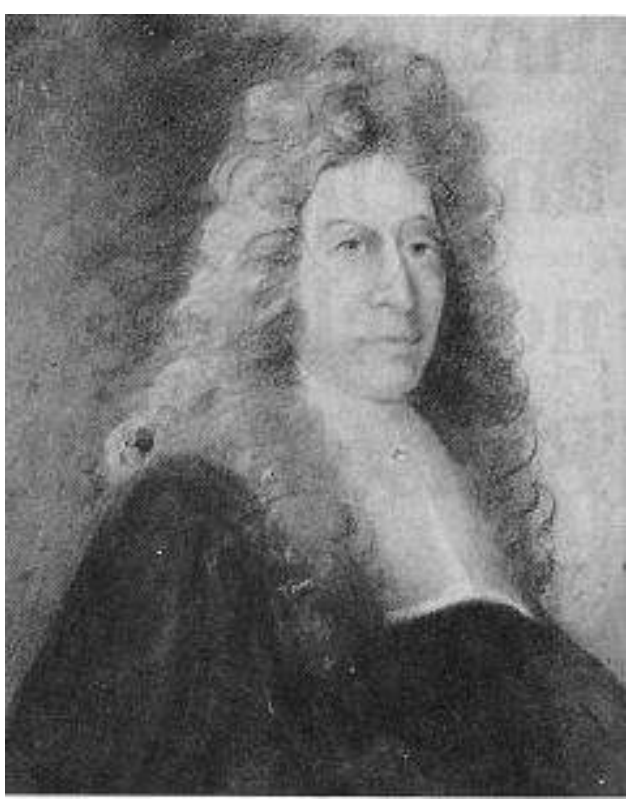

Pastel non signé

Musée de Clermont-Ferrand

\section{Planche III}

\section{Le Graffito de Mirefleurs}




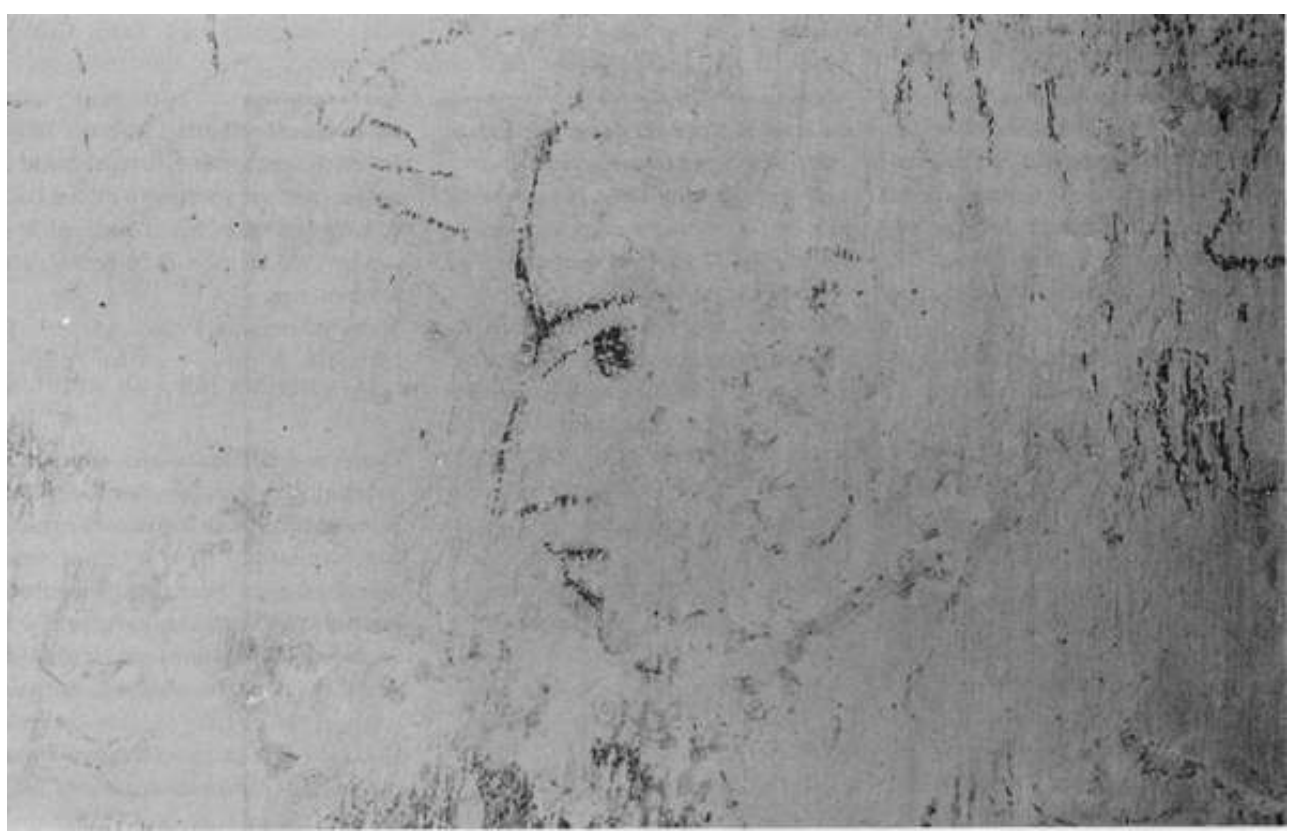

Le village de Mirefleurs est situé sur la rive droite de l'Allier, à dix-huit kilomètres au sud de Clermont-Ferrand.

La maison dite « de Domat » remonte au XV siècle. Elle comporte un rez-de-chaussée et deux étages desservis par un escalier à vis formant une tour. Au sommet de cette tour se trouve une pièce circulaire minuscule de $1 \mathrm{~m} 20$ de diamètre.

13 Cette maison appartenait à Jean Domat, le célèbre jurisconsulte auquel Pascal, en mourant, confia ses papiers, ce qui dit assez bien quels liens d'amitié liaient les deux hommes.

14 La petite pièce du sommet de la tour est couverte d'environ 225 graffiti parmi lesquels le $\mathrm{n}^{\circ} 184 \mathrm{du}$ plan ci-contre présente un personnage de profil qui fait irrésistiblement penser à Blaise Pascal. Ce visage paraît être celui d'un homme jeune entre vingt-cinq et trente ans. Plusieurs traits de physionomie rappellent ceux du portrait dessiné par Domat et du masque mortuaire, les deux témoins iconographiques les moins discutables : le menton est court, les lèvres sont charnues et bien ourlées; la prunelle est plutôt grande ; l'œil est rond et enfoncé dans l'orbite. Le front est haut et bombé. Le nez busqué est cependant plus pointu dans le dessin du mur.

15 Ce graffito pourrait donc être un portrait de Blaise Pascal. D'autant qu'entre mai 1649 et novembre 1650, Blaise Pascal, son père et sa sœur Jacqueline, fuyant les troubles de la Fronde à Paris, ont vécu à Clermont. Pascal en profita pour faire de nouvelles expériences sur le Puy de Dôme, et sa liaison avec Jean Domat était alors familière.

16 Mirefleurs se trouvait à environ une heure et demie d'attelage de Clermont. On peut penser que Domat y invita quelquefois son ami Blaise Pascal. Dès lors, qui pourrait s'étonner de retrouver parmi les familiers croqués sur le mur de la tour, le visage de Blaise Pascal, représenté vers l'âge de 26 ou 27 ans ? (voir Courrier du CIBP n ${ }^{\circ}$ 6). 


\section{Planche IV \\ L'histoire d'un portrait perdu}

17 Les hasards de la trouvaille historique peuvent livrer à la postérité bien des surprises. De celui dont elle tira sa gloire de si bonne heure, la famille Pascal n'aura-t-elle jamais commandé d'autre portrait que posthume? Les pièces venant au jour ont la parole.

Voici, une peinture sur cuivre de $24 \times 20 \mathrm{~cm}$, représentant dans un encadrement ovale peint, de trois quarts regardant sur la droite, un homme jeune aux cheveux milongs. Pascal «ne voulait pas se laisser peindre après sa conversion, mais avant ?... Cela est naturel. Il a l'œil plus heureux et d'un bonheur terrestre. (...) A cette époque il n'était pas si mélancolique. D'ailleurs l'œil sur le tableau est noir et plein de gravité». C'est ainsi qu'en 1923 le possesseur du portrait, Charles Silvestre (1883-1948) décrivait son acquisition à son ami Henri Pourrat. La famille de ce dernier fit don au CIBP de la photographie que nous reproduisons.

19 Le regard est passionné et inquisiteur. Le nez, gros et busqué, se montre ; le menton est relevé ; la lèvre est sensuelle. Tout respire un orgueil légitime.

Un spécialiste des portraits du XVII ${ }^{e}$ siècle, non prévenu, nous a dit: C'est Pascal. D'ailleurs Charles Silvestre n'est pas seul à témoigner pour cette identification: la Bibliographie de Blaise Pascal par Albert Maire, t. v, 1927, p. 86, signale ce même portrait. Nous savons que la peinture fut montrée, vers 1890, à Henri Delaborde, directeur du Cabinet des Estampes, au Musée du Louvre, et que tous trois y reconnurent un portrait de Pascal. Nous savons le nom de celui qui le possédait en 1893. Nous savons quelles armoiries s'y trouveront, peintes au verso.

21 Le dernier possesseur connu du tableau fut le frère de Charles Silvestre, l'abbé Robert Silvestre, prêtre du diocèse de Limoges, qui a été aumônier à l'Hôtel-Dieu de Paris de 1930 à 1963. Il est décédé le 9 janvier 1963, ne laissant en héritage absolument rien.

Mais il connaissait la valeur, spirituelle et historique, de cet objet. À qui l'aura-t-il confié?

Depuis une dizaine d'années nous avons enquêté dans bien des directions, sans aucun succès. Nous demandons maintenant aux médias de donner une large diffusion à notre appel. Cette image bouleversante qui ouvrira un accès autre - et totalement inédit - à la personnalité de Pascal, nul ne peut vouloir la garder pour soi.

(Thérèse Goyet, Directeur adjoint du CIBP)

\section{Planche V \\ Port-Royal, Pascal et l'art de la peinture}

Saint Augustin disait: "Il faut détourner l'esprit des images corporelles.» Car ces images ne sont que le signe visible d'une réalité plus profonde, Dieu, qui seul mérite intérêt.

Les Jansénistes, pour qui la réalité n'était qu'une image, méprisaient naturellement l'image de cette réalité. Ils n'avaient d'autre règle que l'utilité morale. Les tableaux ne pouvaient être que des objets de piété. S'ils toléraient la peinture c'était dans la mesure où elle pouvait avoir valeur d'exemple. Ils n'admettaient donc que les scènes religieuses 
et les portraits de personnage édifiants, capables par leur vie exemplaire «d'inspirer l'amour de la vertu chrétienne » (Lettre de Nicole).

"Je ne puis vous pardonner le vain désir d'avoir mon portrait et je vous dis devant Dieu que je croirais l'offenser mortellement à consentir à ce qu'on me tirât. Est-il possible que vous ne voyiez point la vanité de ce désir et la griève faute que je ferais d'y consentir?» (Lettre de Mère Angélique, Religieuse de Port-Royal).

"Ce jugement », dit Bernard Dorival (Revue des Arts n 3, 1956), « fixe une doctrine qui ne variera pas, puisque, beaucoup plus tard, Nicole devait répéter la même condamnation » :

29 «Il faut donc (...) faire une attention sérieuse sur cette malignité de se vouloir (...) perpétuer en quelque sorte dans un tableau (...) Ne faut-il pas toujours revenir à cette importante vérité : que nous devons faire très peu de cas de la forme extérieure et corruptible où est présentement notre corps. (...) Les personnes de qui l'on souhaite le portrait font fort bien de ne vouloir pas favoriser ce désir. (...) On sait qu'on a des portraits de quelques personnes éminentes par leur sainteté. Mais on sait aussi qu'on leur a comme dérobé leurs portraits par adresse en les faisant peindre sans qu'ils le sussent et que leurs plus intimes amis n'ont jamais pu sur cela obtenir leur consentement. » (lettre conservée à la bibliothèque de la Société de Port-Royal).

Les gens de «Port-Royal n'aiment pas les Beaux-Arts. Ils leur reprochent de détourner la pensée de l'homme de son salut pour l'inviter à la lascivité par le divertissement et le mensonge » (Le Monde de la peinture, $\mathrm{n}^{\circ} 2$, Hachette éditeur).

31 L'Art est un luxe, une vanité et une preuve coupable d'attachement à ce monde, c'est pourquoi «les personnes vraiment chrétiennes «souhaitaient d'être oubliées selon cette vie et cette image terrestre » ( (Nicole, Lettre XCIII, cité par B. Dorival, Revue des Arts $\left.n^{\circ} 3,1956\right)$. M. Bernard Dorival en prend acte : «Comment alors, écrit-il, pouvoir imaginer que Pascal ait pensé sur ce point autrement que les autres Jansénistes ? ... Plus Janséniste que le chef même des Jansénistes,...plus augustinien que Nicole..., comment l'aurait-il été moins dans son attitude face à l'art ? et eût-il pu commander, ou même seulement laissé peindre son portrait? Ceci eût été, à ses yeux, et à tous les yeux de Port-Royal, péché. »

32 En effet, Pascal semble tout à fait acquis au sentiment janséniste : «Quelle vanité que la peinture qui attire l'admiration par la ressemblance des choses, dont on n'admire point les originaux! »(Pascal, Pensées, Lafuma 40/Brunschvicg 134)

33 "Il est injuste qu'on s'attache à moi » (Blaise Pascal). Il semble donc que Pascal, après sa ou ses conversions, devait refuser de laisser faire son portrait, fût-ce par le crayon d'un ami. Mais avant cette époque, pourquoi aurait-il refusé ?

\section{Planche VI \\ Philippe de Champaigne}


Philippe de Champaigne (1602-1674), ex-voto, 1662

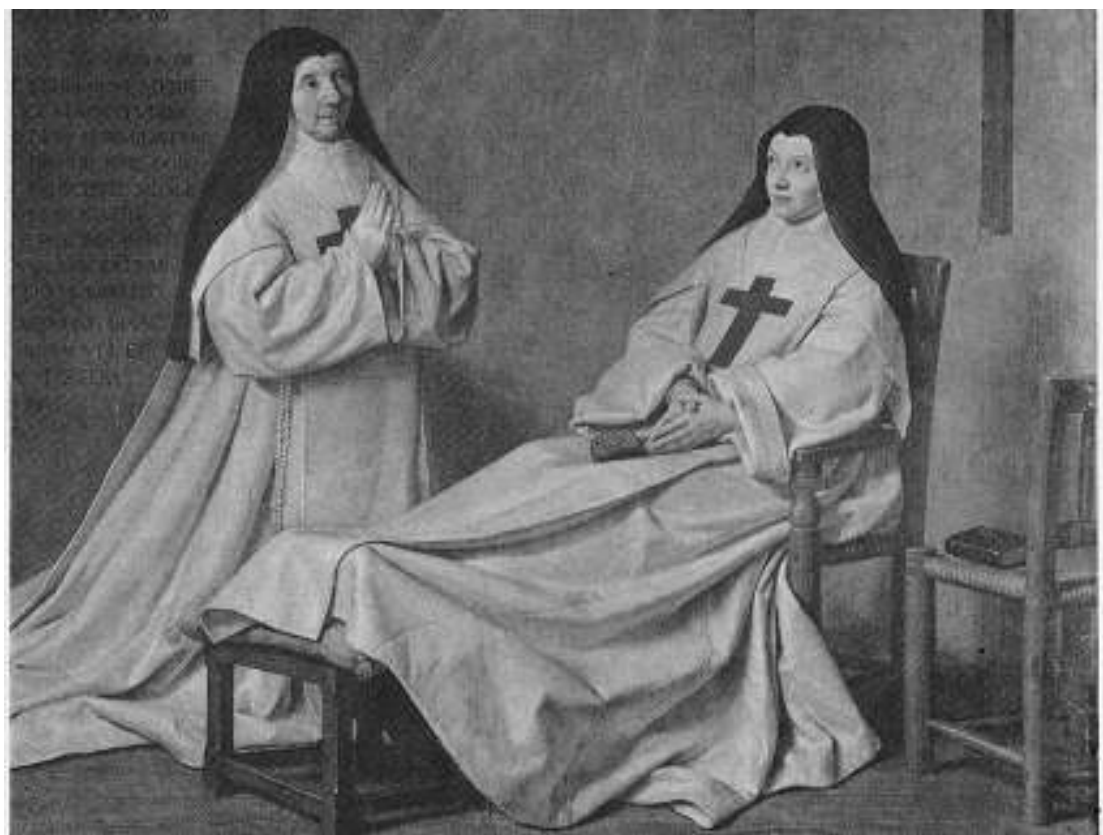

(165 × 229 cm), détail, Paris, musée du Louvre.- CEuvre exécutée pour exprimer sa reconnaissance, après la guérison miraculeuse de sa fille sœur Catherine de sainte Suzanne, les 6 et 7 janvier 1662

C'est surtout pour ses portraits et ses tableaux religieux que Philippe de Champaigne (1602-1674) se rendit célèbre.

D'origine flamande, il connut d'abord les faveurs de Louis XIII, d'Anne d'Autriche, du ministre Richelieu et travailla pour la cour.

L'évolution de sa carrière qui se poursuivit jusqu'au milieu du règne de Louis XIV peut sans doute s'expliquer par deux faits.

En premier lieu, Anne d'Autriche, régente du jeune Louis XIV, tomba sous l'influence du ministre Mazarin. Considéré peut-être comme trop austère et trop critique, Philippe de Champaigne " écarté par une sorte de disgrâce de la petite cour qui gravitait autour de la régente Anne d'Autriche et de Mazarin, avait cherché ailleurs, dès 1646, un succès que ses œuvres religieuses méritaient bien » (Bernard Dorival).

8 D'autre part, il perdit à peu d'intervalle sa femme et plusieurs de ses enfants. "Ces épreuves successives développèrent le mysticisme qui faisait le fonds de sa nature et le tournèrent complètement vers la religion » (Bénézit, Dictionnaire des peintres).

Vers 1647-48 il mit ses deux filles pensionnaires à l'abbaye de Port-Royal et c'est ainsi que des liens s'établirent entre le peintre et les gens de Port-Royal pour lesquels il exécuta des tableaux d'autel, des portraits et des œuvres votives dont la plus célèbre (1662) commémore la guérison miraculeuse de sa fille, sœur Catherine de SainteSuzanne.

Philippe de Champaigne ne pouvait que respecter les idées jansénistes à l'endroit de l'art. Le portrait n'était accepté qu'en raison de l'exemplarité morale du modèle.

S'il peignit "quatorze fois les gens de Port-Royal», ce fut "souvent d'après leurs masques funéraires, quelques fois ad vivum, mais alors généralement à leur insu : jamais leur humilité n'eût consenti à ce qu'on les portraiturât » (B. Dorival, Le Larousse des Grands peintres). 

«Vérité » physique, d'où le réalisme et la perfection scrupuleuse de ses portraits, mais aussi « vérité » psychologique et morale, donnant ainsi une expression plastique de la vie intérieure du sujet.

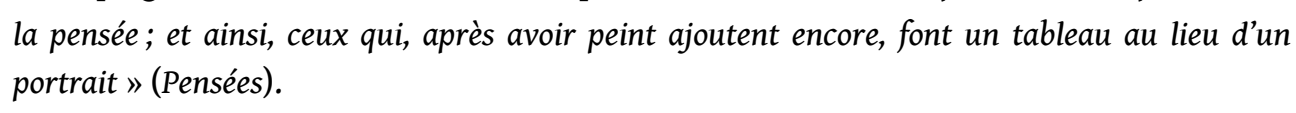
la pensée; et ainsi, ceux qui, après avoir peint ajoutent encore, font un tableau au lieu d'un portrait »(Pensées).

Champaigne allait dans le même sens que Pascal disant : "L'éloquence est une peinture de

44 «Champaigne ne savait pas «ajouter», il fut admirable dans le portrait» (André Hallays).

\section{Planche VII \\ Philippe de Champaigne, peintre de la Cène}

45 Philippe de Champaigne, La Cène, 1648

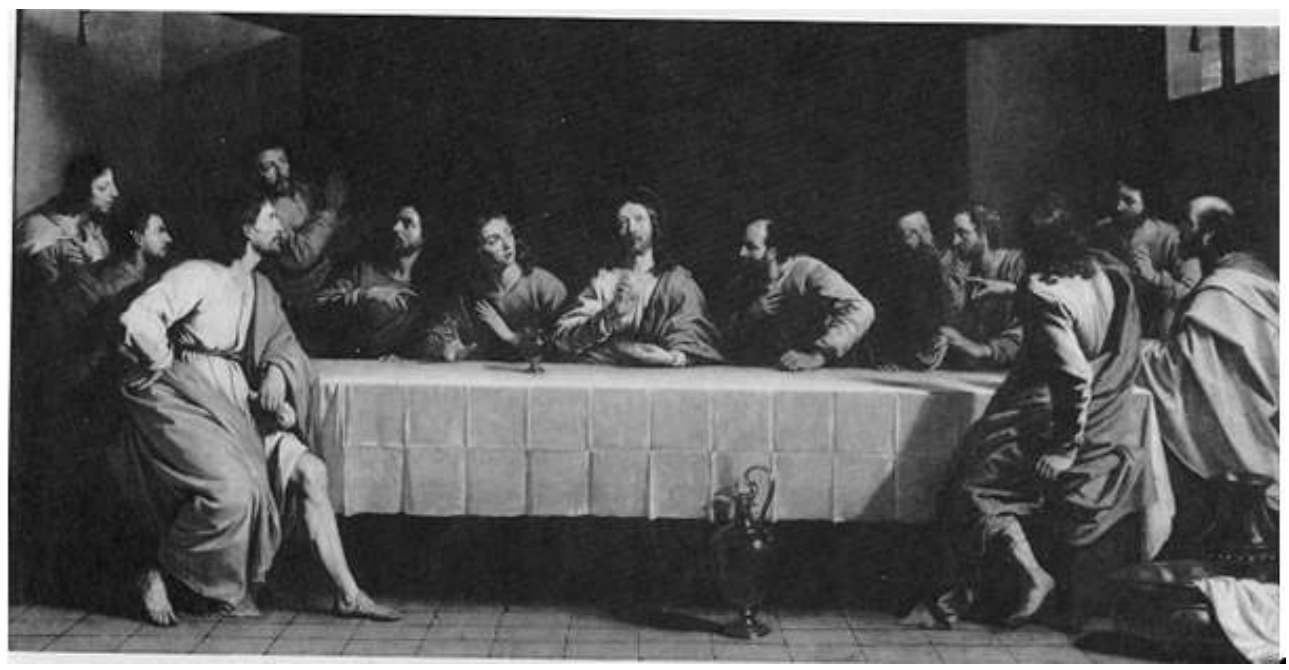

Musée du Louvre, Paris

Philippe de Champaigne peignit en 1648 un tableau de $80 \times 149 \mathrm{~cm}$ pour la chapelle de Port-Royal de Paris, qui est dite « La petite Cène ».

Quatre ans plus tard, en 1652, il exécuta une réplique de 158 x $233 \mathrm{~cm}$ dite « La grande Cène » destinée à Port-Royal des Champs.

Ces tableaux sont tous deux aujourd'hui au Musée du Louvre.

D'après une longue tradition, " toutes les têtes ont été faites d'après nature, et sur des originaux de personnages encore en vie " (Sauval, Histoire de Paris, 1724). En effet, tous les visages de la Cène sont autant de portraits individualisés et non des figures de convention, sauf le visage du Christ, idéalisé pour des raisons évidentes. Car, écrit Bernard Dorival, pour Philippe de Champaigne, seule «la transcription de la réalité (peut) exprimer le contenu spirituel des formes » (Larousse des Grands Peintres).

Le peintre avait donc besoin de douze modèles d'apôtres présentant des visages dont l'expression devait refléter la foi intérieure intense qui animait les premiers disciples du Christ.

51 Où pouvait-il trouver de meilleurs modèles qu'à l'intérieur même de Port-Royal où les hommes étaient si proches de la simplicité et de la pauvreté évangélique?

Courrier du Centre international Blaise Pascal, 13 | 1991 


\section{Planche VIII Philippe de Champaigne, peintre de la Cène}

Mais les Messieurs de Port-Royal n'eussent pas accepté de poser pour un portrait et n'eussent pas davantage apprécié qu'on les jugeât dignes de jouer les apôtres !

Le peintre a donc été forcé de les croquer à leur insu (tous sont de trois quarts ou de puissent s'y reconnaître formellement et lui en faire reproche.

Philippe de Champaigne, La grande Cène, 1652

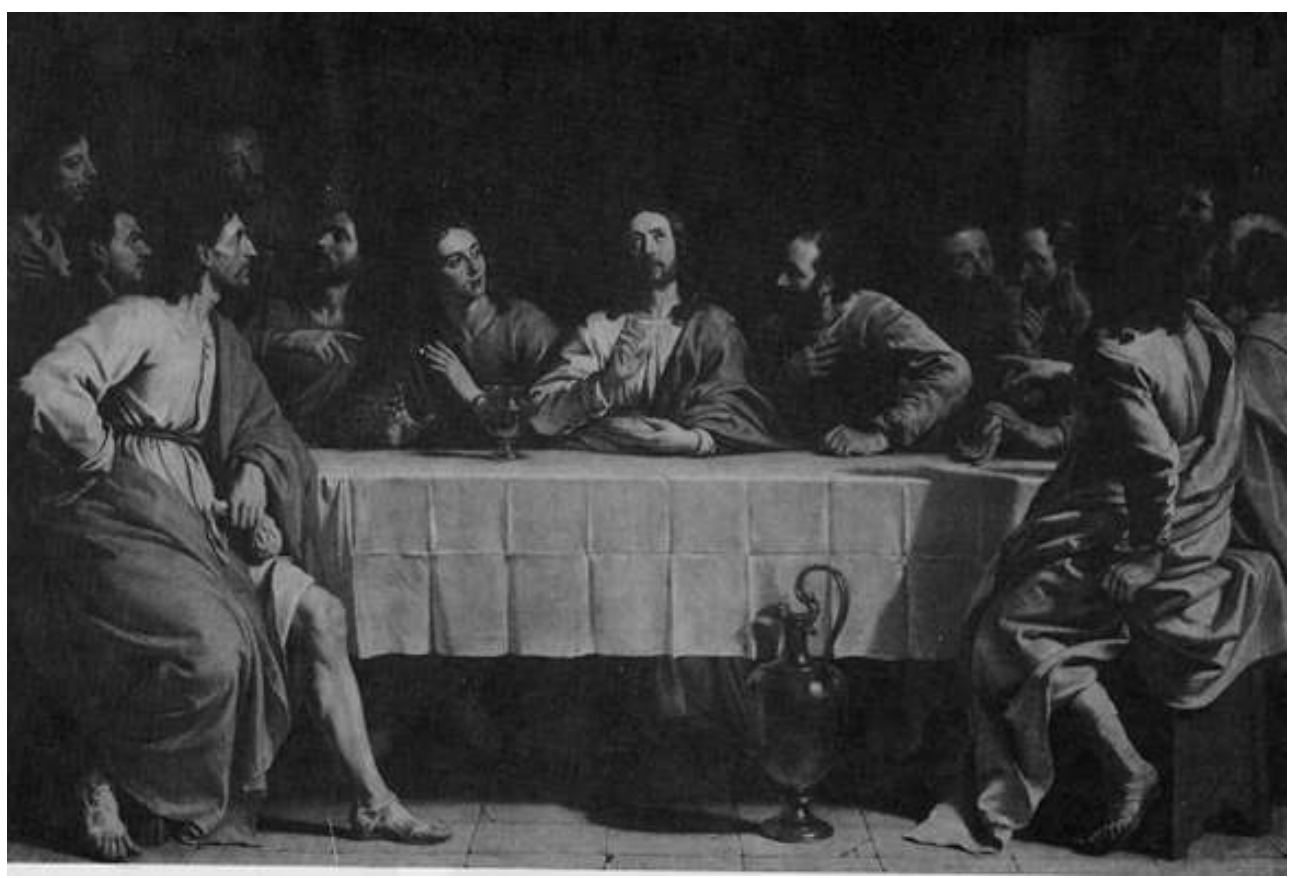

Musée du Louvre, Paris profil). Il s'appliqua ensuite à en modifier suffisamment les traits pour qu'ils ne

La comparaison des deux cènes, peintes par Philippe de Champaigne à quatre ans d'intervalle, met en évidence « son penchant pour l'austérité (...) un goût plus épuré, un ordre plus dépouillé, un classicisme plus sévère" (B. Dorival, Larousse des Grands Peintres).

Dans la seconde version (grande Cène), les couleurs des vêtements sont moins vives. Les détails jugés accessoires ont disparu : Vaisselle en bas à droite, amorce de paysage vu par le peintre, glands de rideaux superflus. L'auréole du Christ elle-même a perdu son clinquant...

\section{Planche IX}

\section{Pascal sous les traits d'un apôtre}

Philippe de Champaigne, détail de la Cène, 1648 


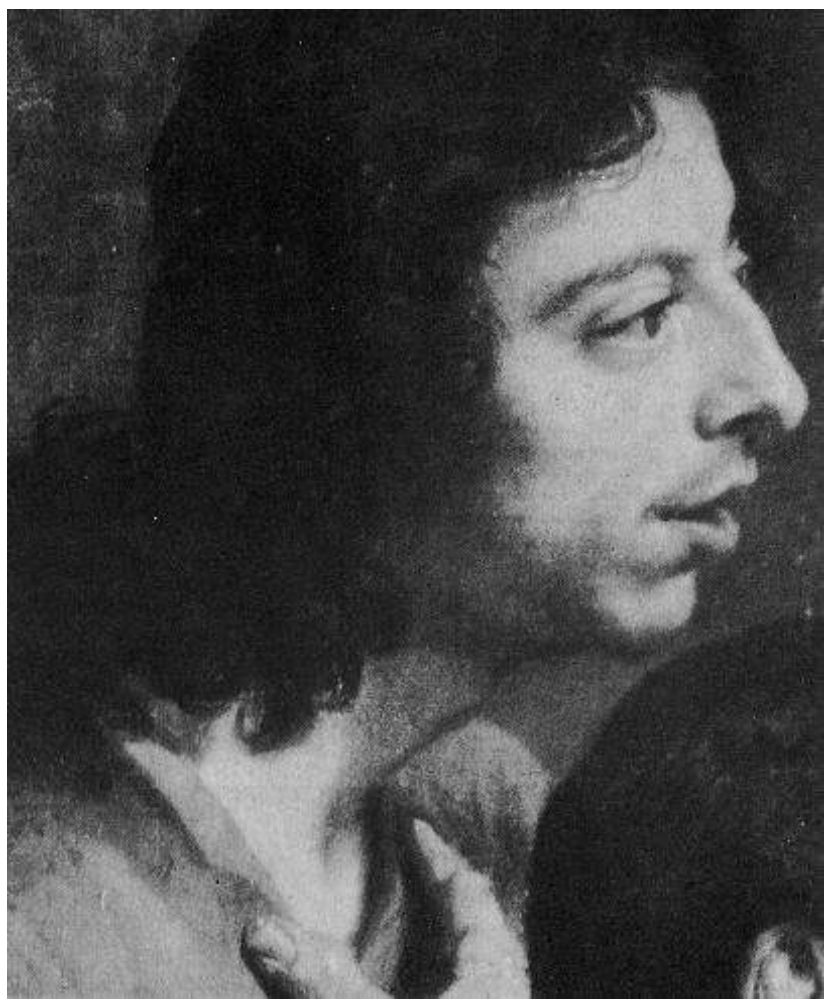

Musée du Louvre, Paris

59 S'il est vrai que Philippe de Champaigne «n'avait aucune raison d'exécuter pour les religieuses un portrait de Pascal » (Jean Mesnard), s'il est vrai également «qu'aucun document ne fournit (...) le moindre indice de relations personnelles entre Philippe de Champaigne et Pascal » (Jean Mesnard), il est par contre tout à fait probable qu'ils se connaissaient, s'étant presqu'en même temps liés avec le milieu janséniste de PortRoyal.

60 Philippe de Champaigne a donc pu « croquer » Pascal, soit à son insu (comme il le fit pour d'autres), soit de mémoire immédiatement après des observations directes : alors que pendant les sermons de M. Singlin, par exemple, Pascal (comme les autres) buvait ses paroles comme cet apôtre boit les paroles du Christ.

61 Lors de l'exécution de la " petite Cène ", Pascal a 25 ans. Lors de la " grande Cène » il en a 29 et la ressemblance y est si flagrante qu'on n'y a jamais reconnu qui que ce soit d'autre que Blaise Pascal. Les traits communs avec les effigies certaines (dessin de Domat et masque mortuaire) y sont nombreux et caractéristiques : profil du nez, forte sous-cloison nasale, pommettes hautes et saillantes, masses charnues de chaque côté de la lèvre inférieure, bouche aux lèvres bien ourlées, coiffure aux cheveux longs et légèrement bouclés, fine moustache...

62 Cet air de famille indiscutable peut en outre se vérifier par comparaison avec le portrait de sa sœur Gilberte. 


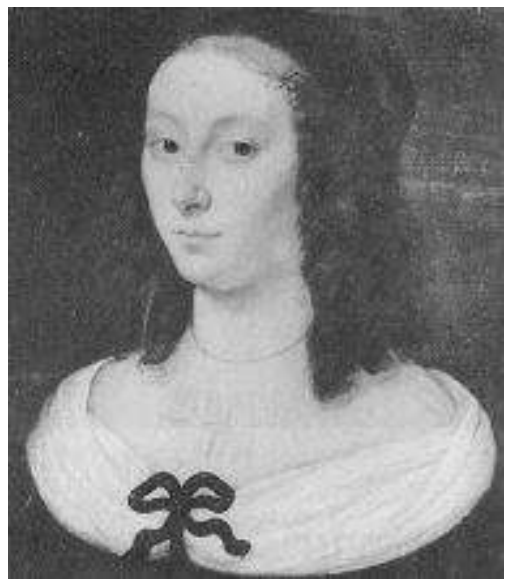

Musée de Clermont-Ferrand, XVII siècle, non signé

\section{Planche X \\ Pourquoi un moulage du visage de Pascal ?}

63 À la mort de Pascal, il n'existait pas de portraits reconnus de sa famille ou de ses amis qui puissent convenir à l'image que les «pascalins » voulaient transmettre de lui : celle du chrétien plutôt que celle du savant.

Le portrait dessiné par Domat avait pu être oublié au fond de quelque tiroir. D'ailleurs il datait de la jeunesse, bien avant que Pascal eût montré les préoccupations religieuses qui le rendront célèbre. Le portrait de la collection Silvestre est peut-être celui d'un Pascal fréquentant les milieux libertins.

La peinture dans la cène de Philippe de Champaigne est un portrait interprété. Il remonte au temps où Pascal poursuit ses travaux scientifiques et n'a pas encore entamé sa carrière de polémiste chrétien...

De plus, la figure est de profil et bouche bée. C'est sans doute à cause de cette insuffisance que la sœur de Pascal, Gilberte, et son mari Florin Périer firent mouler en plâtre son masque mortuaire, dans l'intention de fixer et de diffuser les traits de l'homme, apologiste de la région chrétienne, dont les générations devaient se souvenir et s'inspirer. 

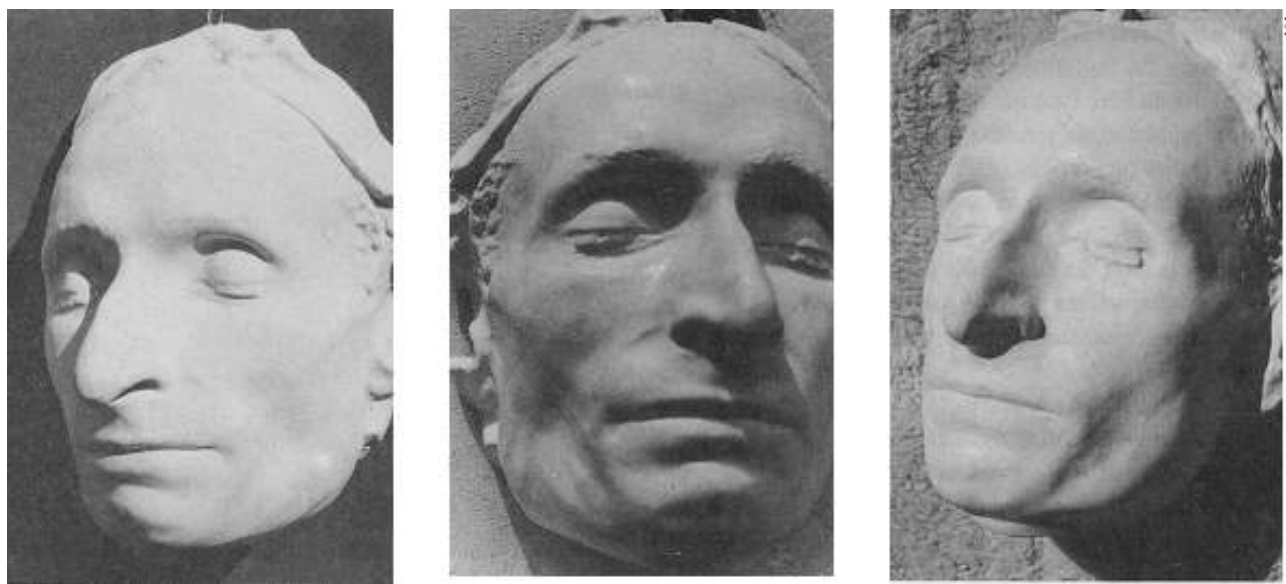

Magny-les-Hameaux, musée de Port-Royal des Champs

\section{Planche XI \\ Le masque mortuaire de Pascal}

Les mouleurs de masques mortuaires étaient des professionnels. Ils connaissaient parfaitement la destination du masque et savaient éviter toute déformation pouvant résulter de leur travail.

Les déformations causées, selon certains ${ }^{2}$, par l'opération du moulage ne sont valables que pour une prise d'empreinte sur modèle vivant. Dans ce cas seulement, comme il y a risque d'étouffement, il convient d'agir très vite et de poser le plâtre en une masse qui écrase alors les chairs molles.

69 Mais, sur un cadavre, les mouleurs procédaient plus lentement. Ils posaient d'abord une mince couche de plâtre qu'ils laissaient s'affermir avant de poser le reste en couche épaisse, évitant ainsi toute altération du modèle. En outre, ils utilisaient comme on le fait encore aujourd'hui un plâtre spécialement préparé pour eux dont le gonflement est pratiquement nul. Pascal, souffrant et amaigri, portant les stigmates d'une longue ascèse, était déjà « méconnaissable » à l'approche de la mort qui dut encore accentuer les déformations de ses traits.

«Au moment de la mort, les yeux se ferment ou s'enfoncent, le nez et la bouche se dépriment dans leurs contours, les joues se tirent, se contournent, enfin une foule de circonstances altèrent la figure, lui enlèvent son caractère et sa physionomie... la froideur, l'empreinte de la mort s'y retrouve malgré tous les soins. » (Encyclopédie Roret, "Mouleur en plâtre», 1910) Ainsi, alors même qu'il est d'une excellente qualité technique ${ }^{3}$, le masque de Blaise Pascal ne nous transmet fidèlement que certains traits de sa physionomie : proportions générales données par l'ossature, angle facial, profil du nez, inclination de l'orifice nasal, haute cloison sous nasale, hauteur du front, pommettes hautes et saillantes, forts globes oculaires. 


\section{Planche XII \\ Le portrait posthume par Quesnel}

Portrait posthume de Pascal par Quesnel

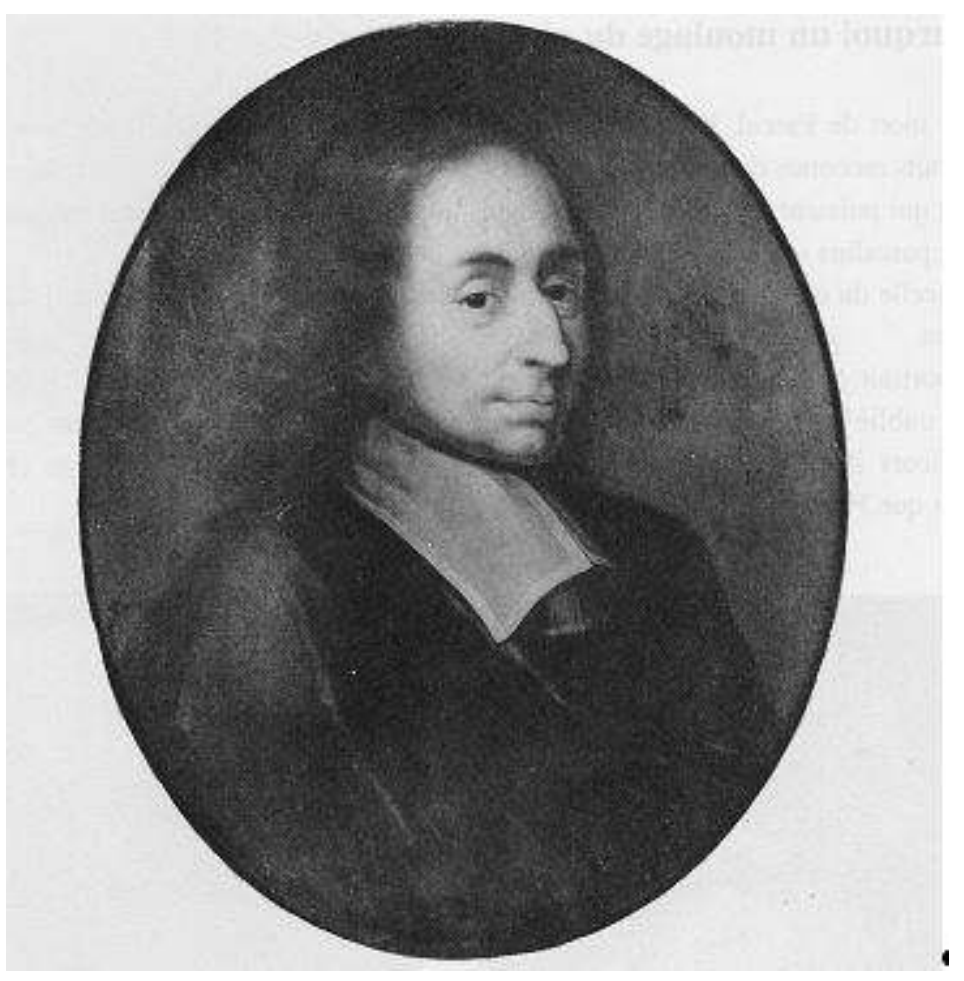

Quand il n'existait aucun portrait connu d'un personnage dont on voulait conserver l'image après son décès, on dessinait un portrait directement d'après le mort. Ou bien, s'il n'y avait pas d'artiste immédiatement disponible, on faisait un moulage en cire ou, le plus souvent, un moulage en plâtre. Et c'est d'après ce plâtre qu'on exécutait plus tard un premier portrait qui serait ensuite recopié ou gravé en plusieurs exemplaires pour être diffusé.

La famille de Pascal s'adressa à un peintre modeste ami de la famille, François Quesnel, frère du Père Pasquier Quesnel. Le masque apportait de précieux renseignements sur les proportions générales et l'architecture de la tête. Mais on savait que le visage était altéré par la maigreur, la souffrance et la mort. La famille dut donc fournir les informations indispensables pour corriger les déformations et le peintre fit ce qu'il put...

Réussit-il à le faire bien ressemblant? La famille et les amis du défunt n'osaient se prononcer: "Quand M. Quesnel (...) eut fait le portrait de M. Pascal, qui était mort depuis plusieurs années, on montra ce portrait à un grand nombre de personnes qui avaient connu ce grand homme [...] l'horloger du coin s'étant écrié: c'est bien ce monsieur qui avait sa montre attachée à son poignet, ce témoignage spontané suffit à (les) rassurer pleinement » (raconté par Marguerite Périer au Père Guerrier). Le portrait original peint par François Quesnel aurait disparu. ("Blaise Pascal Auvergnat", La famille à l'œuvre, musée de ClermontFerrand, 1981, $\mathrm{n}^{\circ}$ 59). 
74 Quoiqu'il en soit, le portrait qui est tenu pour l'original (Exposition Blaise Pascal, Bibliothèque Nationale, 1962, $n^{\circ} 581$ ) vient du Père Guerrier; il appartenait à la baronne de Romagnat, puis au marquis Doria (vers 1910) et il est encore aujourd'hui propriété de la descendance.

75 Nous devons la photographie ici présentée (photographie de l'original ou de la première copie d'après l'original) à l'obligeance de son actuel propriétaire.

76 Les copies de ce portrait ou d'après ce portrait, inversées ou non et plus ou moins fidèles, sont innombrables. Car rien n'empêche les artistes de tous les temps de réanimer cette image de Pascal ou celle du destin de Domat...

\section{Planche XIII \\ Quelques portraits}

Portrait de Pascal

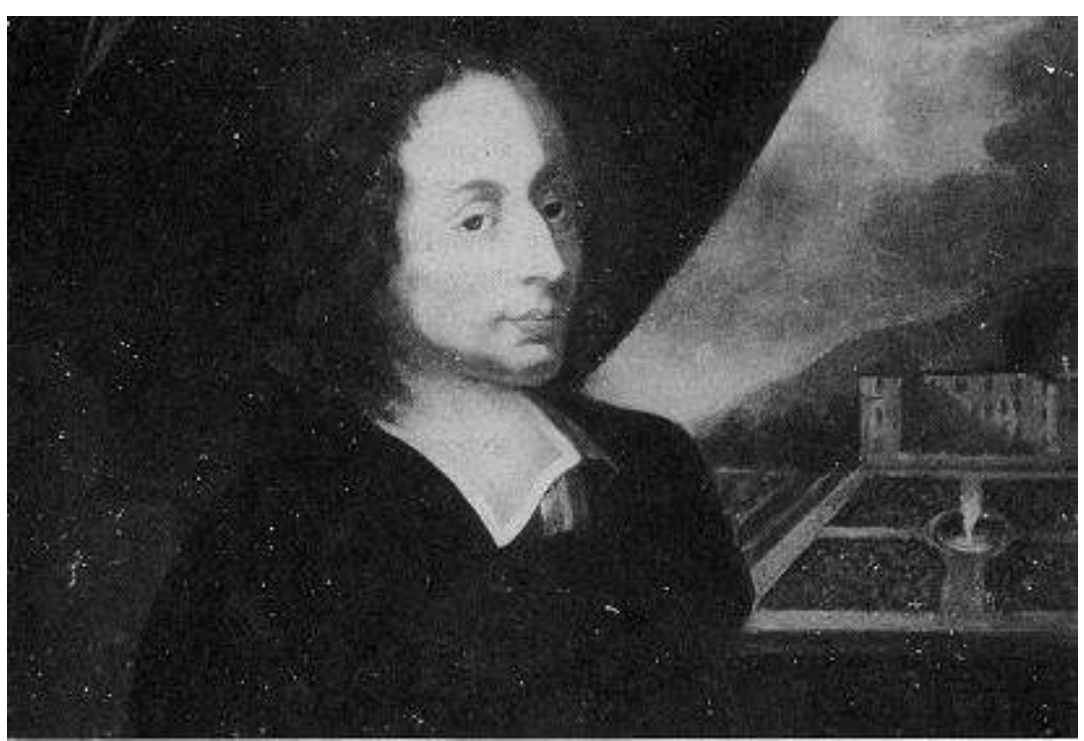




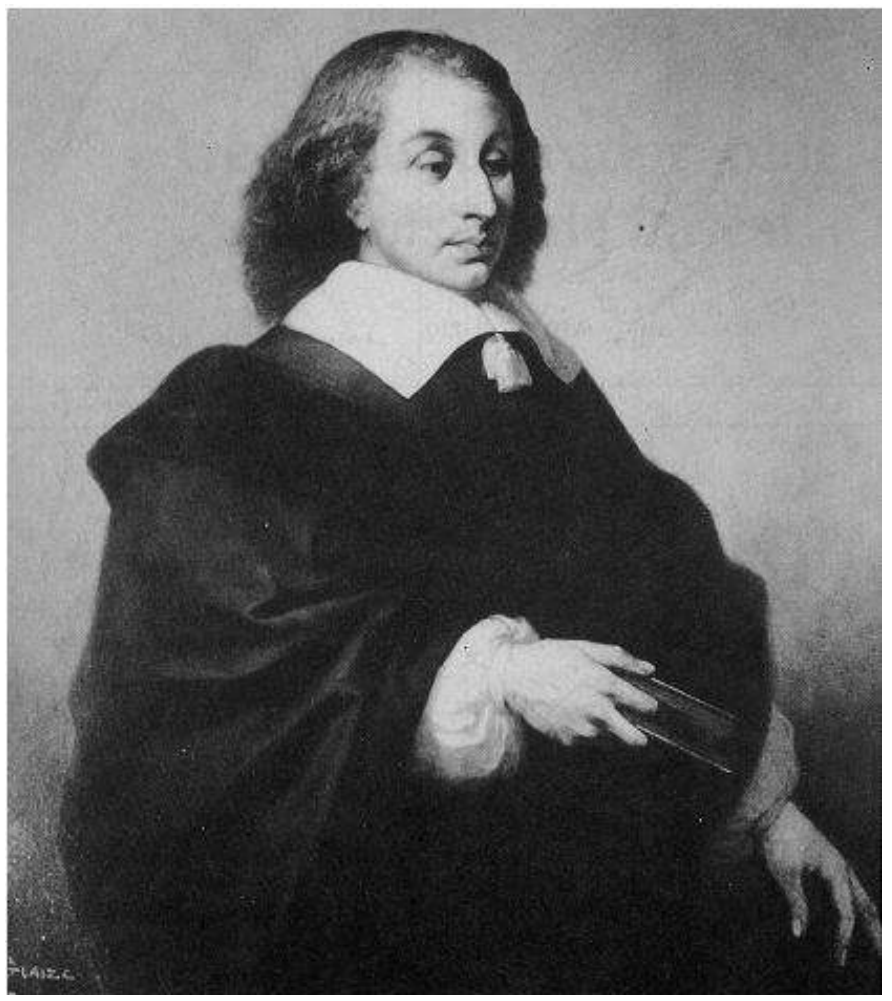

\section{Planche XIV}

\section{La gravure d'Edelinck et ses dérivés}

77 Edelinck grava le portrait de Pascal d'après le portrait posthume que François Quesnel avait lui-même exécuté d'après le masque mortuaire.

78 La date de la première gravure, relativement tardive (1692) après la mort de Pascal (1662), peut s'expliquer par le fait que « le tableau de Quesnel était simplement destiné à la famille... C'est, au contraire, au grand public que s'adressait la gravure d'Edelinck. Elle n'avait aucune raison d'être tant que la gloire de Pascal ne s'était pas étendue... » (J. Mesnard, Blaise Pascal. CEuvres complètes, 1964).

79 L'exemplaire présenté ici précise la nature de l'original utilisé par l'artiste : QVL delin (Quesnel delineavit).

80 «C'est d'un dessin que s'est inspiré le graveur, non d'une peinture ».

81 « Le tableau était à Clermont. Edelinck travaillait à Paris. Le dessin dont il disposa était sans doute une esquisse préparatoire au tableau. »

82 "C'est elle (la gravure), et ses nombreux exemplaires qui ont, beaucoup plus que les toiles enfouies dans les collections particulières, fait connaître au grand public les traits de Pascal. Elle a servi de modèle à d'autres graveurs, à des statuaires, à des graveurs en médailles, et aussi à de nombreux peintres. » (J. Mesnard, Ibid.). 
Gravure d'Edelink représentant Pascal

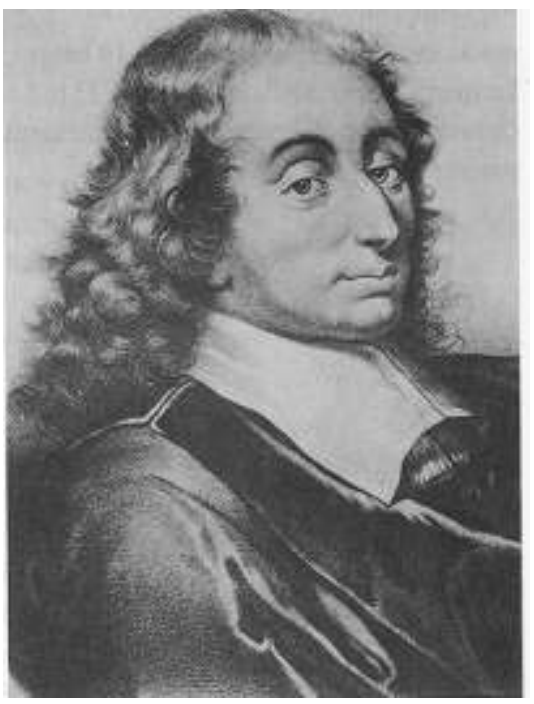

L'exemplaire présenté ici précise la nature de l'original utilisé par l'artiste : QVL delin (Quesnel delineavit).

\section{Planche XV}

\section{Les dessins d'Yves Morvan}

Dessins réalisés par Yves Morvan
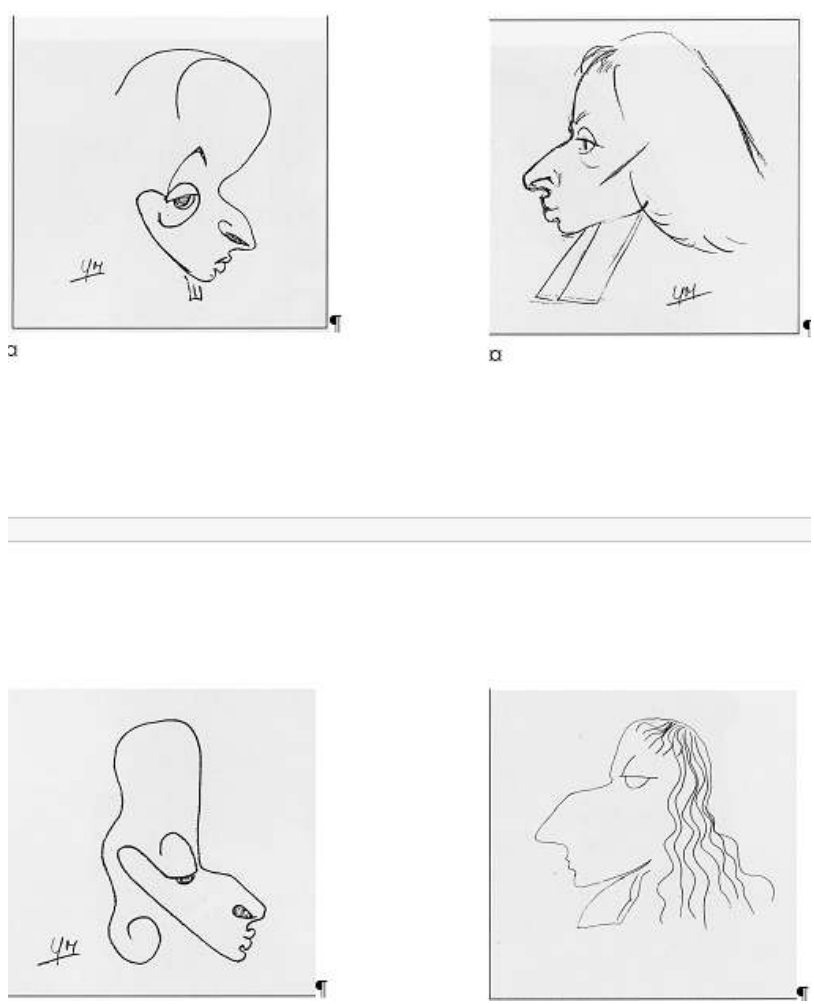

Dessin d'élève

Dessins présentés lors de la semaine des Arts (29 mai- 5 juin 1988) au Lycée et Collège Blaise Pascal de Clermont-Ferrand. 


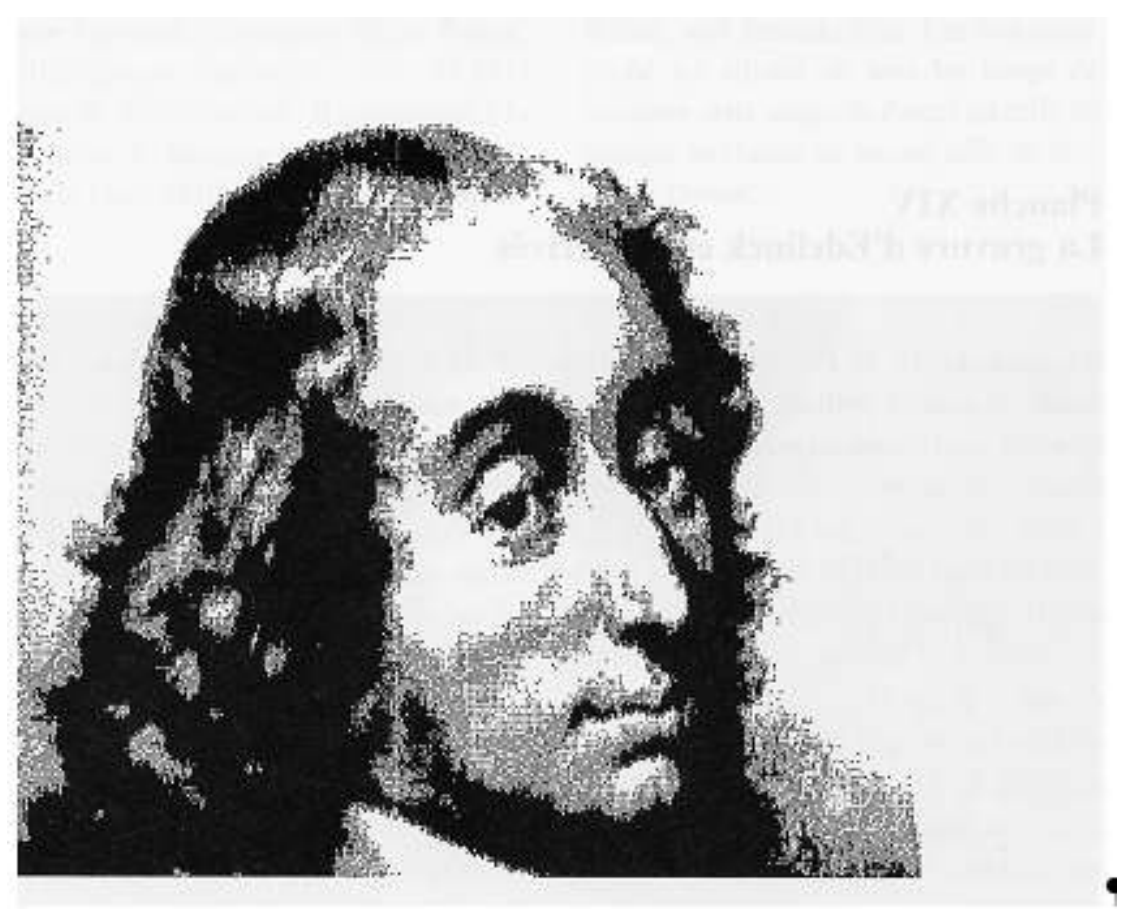

Réalisé par le Club Informatique MicrosData en 1990.

\section{NOTES}

1. Sur ce point de la valeur de témoignage direct à attribuer au dessin de Domat, nous nous permettons de renvoyer globalement à notre étude " Pascal d'après nature ", dans le Bulletin historique et Scientifique de l'Auvergne, janvierjuin 1987 (publication de l'Académie des Sciences, Belles Lettres et Arts de Clermont Ferrand.)

2. Après Ulysse Moussalli, Bernard Dorival (Revue des Arts $n^{\circ} 3$, 1956) et Jean Mesnard («Blaise Pascal ", Euvres complètes, t. 1, 1964) font état de déformations dues «à la double action de la charge du plâtre et de sa dilatation » (Moussalli, Le vrai visage de Blaise Pascal, Paris, 1952).

3. Le masque original (première épreuve sortie du moule en creux) a disparu ainsi que ce moule lui-même. Les nombreux masques existants sont des surmoulages, plus ou moins précis, réalisés à partir du masque original. 